\title{
Controller Design for Delay Margin Improvement
}

\author{
A. N. Gündeş ${ }^{\dagger}$
}

\begin{abstract}
Two important design objectives in feedback control are steady-state error minimization and delay margin maximization. In general these two objectives work against each other. This paper starts with an initial controller designed to satisfy the steady-state error requirement, and shows how one can modify it to improve the delay margin without changing the steady-state error.
\end{abstract}

\section{INTRODUCTION}

In feedback control theory, one of the most important stability robustness measures is the delay margin $(D M)$. Classical control techniques, such as lead-lag and PID controller designs, try to meet a given desired phase margin requirement, [1], [2]; but these do not directly guarantee the amount of delay uncertainty that can be tolerated by such designs. In order to tackle this issue directly, many studies in recent years have been devoted to the delay margin optimization problem, [5], [9], [10]. More precisely, for a given nominal plant we would like to compute the largest possible $D M$ one can obtain over all stabilizing controllers. Finding the optimal controller maximizing the delay margin is still an open problem for the general class of unstable plants with multiple poles in the right half plane, [10]. Therefore, recent publications on this topic consider some special class of plants, or investigate upper and lower bounds of the largest achievable delay margin, see e.g. [4], [5], [9].

In this paper we consider the delay margin improvement problem, over an initially designed stabilizing controller, which takes care of the steady state error requirement. This is similar to lead-lag controller design, where one modifies an initially designed controller to improve the phase margin. For stable plants, typically lag controllers increase the phase margin and decrease the crossover frequency, [1]. Hence, for such systems the delay margin can be improved by lag controllers. However, for unstable plants delay margin improvement is not as simple.

The paper is organized as follows. In Section II the delay margin (DM) is defined; then, its computation and lower bounds are discussed. The trade-off with DM maximization and tracking error minimization is also illustrated. In Section III it is assumed that an initial controller is designed to make the steady-state error zero when unit step reference input is applied. Then, a new method is proposed to modify this controller to improve the delay margin without changing

\footnotetext{
${ }^{\dagger}$ A. N. Gündes is with the Department of Electrical and Computer Engineering, University of California, Davis, CA 95616, USA angundes @ucdavis.edu

${ }^{\ddagger} \mathrm{H}$. Özbay is with the Department of Electrical and Electronics Engineering, Bilkent University, Ankara 06800, Turkey hitayebilkent.edu.tr
}

\author{
H. Özbay
}

the poles of the controller at $s=0$. Conclusions and a discussion on future directions can be found in the last section. This brief version of the paper outlines the proposed method for controller design for DM improvement without technical details. For proofs and and further discussion with more illustrative examples we refer to the full version, [3].

Notation: The closed right-half-plane (RHP) is $\mathbb{C}_{+}=\{s \in$ $\mathbb{C} \mid \Re e(s) \geq 0\}$, and the open left-half-plane (LHP) is $\mathbb{C}_{-}=\{s \in \mathbb{C} \mid \Re e(s)<0\}$. The region of instability $\mathcal{U}$ is the extended closed RHP, i.e., $\mathcal{U}=\mathbb{C}_{+} \cup\{\infty\}$. Real and positive real numbers are denoted by $\mathbb{R}$ and $\mathbb{R}_{+}$, respectively; $\mathcal{R}_{p}$ denotes real proper rational functions of $s ; \mathcal{S} \subset \mathcal{R}_{p}$ is the stable subset with no poles in $\mathcal{U}$. The space $\mathcal{H}_{\infty}$ is the set of all bounded analytic functions in $\mathbb{C}_{+}$. A matrix-valued function $H$ is in $\mathcal{M}\left(\mathcal{H}_{\infty}\right)$ if all its entries are in $\mathcal{H}_{\infty}$. For $f \in \mathcal{H}_{\infty}$, the norm $\|\cdot\|$ is defined

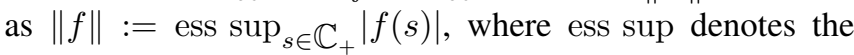
essential supremum. The degree of the polynomial $d$ is denoted by $\delta(d)$. For simplicity, we drop $(s)$ in transfer functions such as $\mathbf{P}(s)$ when this causes no confusion.

\section{PROBLEM DEFINITION AND PRELIMINARY REMARKS}

Consider the feedback system $\mathscr{S}\left(e^{-s h} \mathbf{P}, \mathbf{C}\right)$ in Fig. 1 . The rational transfer function $\mathbf{P} \in \mathcal{R}_{p}$ represents a given nominal plant (without time delays), and $\mathbf{C} \in \mathcal{R}_{p}$ is the rational transfer function of the controller. With $u, v, w, y$ as inputs and outputs, the closed-loop map $\mathbf{H}$ is

$$
\mathbf{H}=\left[\begin{array}{cc}
\mathbf{C} H_{e u} & -\mathbf{C} H_{e u} e^{-s h} \mathbf{P} \\
H_{y u} & H_{e u} e^{-s h} \mathbf{P}
\end{array}\right]
$$

where the input-output map from $u$ to $y$ (complementary sensitivity function) is denoted by $H_{y u}$; the input-error map from $u$ to $e$ (sensitivity function) is denoted by $H_{e u}$ :

$$
\begin{aligned}
H_{y u} & =e^{-s h} \mathbf{P C}\left(1+e^{-s h} \mathbf{P C}\right)^{-1} \\
H_{e u} & =\left(1+e^{-s h} \mathbf{P C}\right)^{-1}=I-H_{y u} .
\end{aligned}
$$

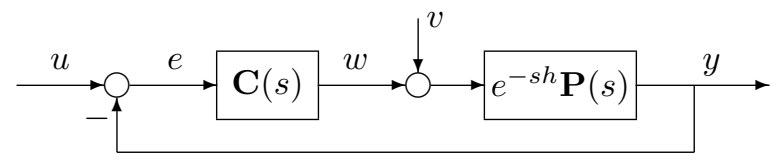

Fig. 1. The feedback system $\mathscr{S}\left(e^{-s h} \mathbf{P}, \mathbf{C}\right)$.

Definition 1: a) The feedback system $\mathscr{S}\left(e^{-s h} \mathbf{P}, \mathbf{C}\right)$ shown in Fig. 1 is stable if $\mathbf{H}$ is in $\mathcal{M}\left(\mathcal{H}_{\infty}\right)$.

b) The controller $\mathbf{C} \in \mathcal{R}_{p}$ is a stabilizing controller for $e^{-s h} \mathbf{P}$ if $\mathscr{S}\left(e^{-s h} \mathbf{P}, \mathbf{C}\right)$ is stable. 
c) The system $\mathscr{S}\left(e^{-s h} \mathbf{P}, \mathbf{C}\right)$ is stable and has integral-action if the closed-loop map $\mathbf{H}$ is stable, and the (input-error) transfer function $H_{e u}$ has zeros at $s=0$.

d) The controller $\mathbf{C}$ is an integral-action controller if $\mathbf{C}$ stabilizes $e^{-s h} \mathbf{P}$ and $\mathbf{C}$ has at least one pole at $s=0$.

e) Let $\mathbf{C} \in \mathcal{R}_{p}$ be a stabilizing controller for the delayfree plant $\mathbf{P}$. The minimum time-delay $h_{m}>0$ such that the closed-loop system $\mathscr{S}\left(e^{-s h_{m}} \mathbf{P}, \mathbf{C}\right)$ becomes unstable is called the delay margin (DM).

An initial controller $\mathbf{C}_{o}(s)$ is designed to stabilize the delay-free feedback system $\mathscr{S}\left(\mathbf{P}, \mathbf{C}_{o}\right)$. The input-output transfer function $H_{y u}$ with the controller $\mathbf{C}_{o}$ is defined as

$$
H_{o}:=\mathbf{P C}_{o}\left(1+\mathbf{P C}_{o}\right)^{-1} \text {. }
$$

Assumption. Throughout the paper we assume that the stabilizing controller $\mathbf{C}_{o}$ is designed so that the open-loop system $G_{o}=\mathbf{P C}_{o}$ is strictly proper. Consequently, the closed-loop transfer function $H_{O}$ is also strictly proper. This design makes sure that the characteristic equation of the feedback system is a retarded quasi-polynomial. Hence we do not have to worry about the possibility of neutral chain of poles asymptotically approaching a vertical line in $\mathbb{C}$.

By continuity, the feedback system $\mathscr{S}\left(e^{-h s} \mathbf{P}, \mathbf{C}_{o}\right)$ with delayed plant is stable for all $h \in\left[0, h_{m}\right)$ for some $h_{m}>0$. The largest possible $h_{m}$ satisfying this property is the delay margin $(D M)$ of the feedback system $\mathscr{S}\left(\mathbf{P}, \mathbf{C}_{o}\right)$.

In what follows we use the delay margin lower bound determined from

$$
D M>\left\|s H_{o}\right\|^{-1}
$$

which can be computed easily from the $\mathcal{H}_{\infty}$-norm of a rational transfer function (the related Matlab command is hinfnorm). It should be also noted that for any rational minimum phase transfer function $w_{h}(s)$ satisfying

$$
\left|w_{h}(j \omega)\right| \geq \psi_{h}(\omega), \quad \forall \omega, \quad \forall h>0,
$$

a lower bound of the delay margin is given by the largest $h>0$ that satisfies

$$
\left\|w_{h} H_{o}\right\|<1
$$

Obviously $w_{h}(s)=h s$ is a special case that satisfies (5). Various possible choices of $w_{h}(s)$ can be found in [8], [10]. Thus, once the controller $\mathbf{C}_{o}$ is free in $H_{o}$, a lower bound of the largest achievable delay margin can be computed by solving a Nevanlinna-Pick interpolation problem resulting from (5) (see Theorem 4.4 of [10], and also Section 5.1.2 of [8]). However, the controller obtained from this design may have poor step response performance. As an example, consider a simple case where $\mathbf{P}(s)=(s+a) /(s-p)$, with $a>0$ and $p>0$. This corresponds to a single interpolation condition and a lower bound of the delay margin is the largest $h>0$ satisfying $\left|w_{h}(p)\right|<1$ with the corresponding optimal controller

$$
\mathbf{C}_{o p t}(s)=\frac{w_{h}(p)}{(s+a)}\left(\frac{(s-p)}{w_{h}(s)-w_{h}(p)}\right) .
$$

This controller, which is designed to maximize a lower bound of the delay margin, typically does not have high gain at low frequencies; hence it will lead to a large steadystate error $e_{\mathrm{Ss}}$ for step-like reference inputs, where $e_{\mathrm{SS}}=$ $\lim _{s \rightarrow 0}\left(1-\frac{w_{h}(p)}{w_{h}(s)}\right)$. Note that the steady-state error is nonzero whenever $w_{h}(0) \neq w_{h}(p)$. Typically, $w_{h}$ is chosen to have very small values at $s=0$, so this means that the steady-state error gets large as $p$ gets large.

\section{DELAY MARGIN IMPROVEMENT}

\section{A. Delay Margin Improvement for Stable Plants}

Proposition 1: Let $\mathbf{P} \in \mathcal{S}$. Let $\mathbf{C}_{o}$ be a controller that stabilizes $\mathbf{P}$, i.e., for $\tilde{Q} \in \mathcal{S}$, let

$$
\mathbf{C}_{o}=\tilde{Q}(1-\mathbf{P} \tilde{Q})^{-1} \text {. }
$$

In order to satisfy the standing Assumption stated above, the following restriction is imposed on $\tilde{Q}$.

a) In (6), for any $Q \in \mathcal{S}$ and $a \in \mathbb{R}_{+}$, let $\tilde{Q} \in \mathcal{S}$ be such that $\left(\mathbf{P C}_{o}\right)(\infty)=0$, i.e., let

$$
\tilde{Q}:= \begin{cases}Q, & \text { if } \mathbf{P}(\infty)=0 \\ \frac{1}{s+a} Q, & \text { if } \mathbf{P}(\infty) \neq 0\end{cases}
$$

i) Let the controller $\mathbf{C}_{o}$ in (6) be pre-specified, i.e., $\tilde{Q} \in \mathcal{S}$ is fixed. A lower bound on the delay margin is given by $\tau_{m}$ :

$$
\tau_{m}=\|s \mathbf{P} \tilde{Q}\|^{-1} \text {. }
$$

The controller $\mathbf{C}_{o}$ in (6) stabilizes $e^{-s h} \mathbf{P}$ for all $h \in\left[0, \tau_{m}\right)$. ii) For a given delay $h=\tau \in \mathbb{R}_{+}$, the controller $\mathbf{C}_{o}$ in (6) can be designed to stabilize $e^{-s \tau} \mathbf{P}$ by choosing $Q \in \mathcal{S}$ in (7) such that

$$
\|Q\|< \begin{cases}\tau^{-1}\|s \mathbf{P}\|^{-1}, & \text { if } \mathbf{P}(\infty)=0 \\ \tau^{-1}\left\|\frac{s}{s+a} \mathbf{P}\right\|^{-1}, & \text { if } \mathbf{P}(\infty) \neq 0\end{cases}
$$

This means that arbitrarily large delay margin can be achieved by the controller choice determined via (9).

b) Integral-action controllers: Assume that $\mathbf{P}(0) \neq 0$. For any $Q_{I} \in \mathcal{S}$ and $a, b \in \mathbb{R}_{+}$define

$$
\tilde{Q}_{I}:=\frac{b}{s+b} \mathbf{P}(0)^{-1}\left(1+\frac{s}{(s+a)} Q_{I}\right) .
$$

With $\tilde{Q}_{I}$ as in (10), the controller $\mathbf{C}_{I}$ given by (11) is an integral-action controller that stabilizes $\mathbf{P}$ :

$$
\mathbf{C}_{I}=\tilde{Q}_{I}\left(1-\mathbf{P} \tilde{Q}_{I}\right)^{-1} .
$$

i) Let the controller $\mathbf{C}_{I}$ in (11) be pre-specified, i.e., $\tilde{Q}_{I} \in \mathcal{S}$ in (10) is fixed. A lower bound on the delay margin is given by $\tau_{m I}$ defined as

$$
\tau_{m I}=\frac{1}{b}|\mathbf{P}(0)|\left\|\frac{s}{(s+b)} \mathbf{P}\left(1+\frac{s}{(s+a)} Q_{I}\right)\right\|^{-1} .
$$

The controller $\mathbf{C}_{I}$ in (11) stabilizes $e^{-s h} \mathbf{P}$ for all $h \in$ $\left[0, \tau_{m I}\right)$.

ii) For a given delay $h=\tau \in \mathbb{R}_{+}$, the controller $\mathbf{C}_{I}$ in (11) can be designed to stabilize $e^{-s \tau} \mathbf{P}$ by choosing any $Q_{I} \in \mathcal{S}$, and $b>0$ in (10) such that

$$
0<b<\frac{1}{\tau}|\mathbf{P}(0)|\left\|\mathbf{P}\left(1+\frac{s}{(s+a)} Q_{I}\right)\right\|^{-1} .
$$


Furthermore, once $b$ is chosen as in (13), the corresponding lower bound $\tau_{m I}$ can be found as in (12), and $\tau_{m I} \geq \tau$. Therefore, the controller $\mathbf{C}_{I}$ in (11) also stabilizes $e^{-s h} \mathbf{P}$ for all $h \in\left[0, \tau_{m I}\right)$, where $\tau_{m I} \geq \tau$.

Example 1: For $\mathbf{P} \in \mathcal{S}$ given in (14), since $\mathbf{P}(0) \neq 0$, it is possible to design integral-action controllers as in (11):

$$
P=\frac{\left(s^{2}-8 s+20\right)}{(s+3)(s+4)} \text {. }
$$

Choosing $Q_{I}=0$ and $b=0.9$, with $|\mathbf{P}(0)|^{-1}=0.6$, the integral-action controller in (11) and the corresponding delay-free closed-loop transfer function $H_{y u}$ become

$$
\begin{aligned}
\mathbf{C}_{I} & =\frac{10.8(s+3)(s+4)}{s\left(20 s^{2}+147.2 s+452.4\right)} \\
H_{y u} & =\frac{0.54\left(s^{2}-8 s+20\right)}{(s+0.9)(s+3)(s+4)} .
\end{aligned}
$$

i) By (12), $\tau_{m I}$ is

$$
\tau_{m I}=\frac{1}{b} \mathbf{P}(0)\left\|\frac{s}{(s+b)} \mathbf{P}\right\|^{-1}=1.4371 .
$$

Then the controller $\mathbf{C}_{I}$ in (15) stabilizes $e^{-s h} \mathbf{P}$ for all $h \in$ $\left[0, \tau_{m I}\right)$. The exact delay margin is $2.5481 \mathrm{~s}$.

ii) For a fixed $\tau>0,\|P\|=P(0)$ implies that (13) is satisfied for $0<b<\tau^{-1}$. For example, suppose that $\tau=2$; then (13) is satisfied for $0<b<0.5$. The choice of $b<1$ then determines $\tau_{m I}$, and the controller $\mathbf{C}_{I}$ in (15) stabilizes $e^{-s h} \mathbf{P}$ for all $h \in\left[0, \tau_{m I}\right)$. For example,

$$
b=0.5 \text { gives } \tau_{m I}=2.3132>\tau,
$$

and

$$
b=0.2 \text { gives } \tau_{m I}=5.3366>\tau .
$$

Small values of $b$ makes the lower bound of the delay margin large, but this leads to a slower step response. This is a fundamental trade-off in controller design.

\section{B. Delay Margin Improvement for Unstable Plants}

Consider an unstable plant whose transfer function $\mathbf{P}$ is factored into numerator and denominator polynomials as

$$
\mathbf{P}(s)=\frac{n(s)}{d_{s}(s) d(s)} .
$$

The roots of $d$ and $d_{s}$ are the $\mathbb{C}_{+}$-poles and the $\mathbb{C}_{-}$-poles of $\mathbf{P}$, respectively, and $d$ is a monic polynomial. Since $\mathbf{P}$ is unstable, it has at least one $\mathbb{C}_{+}$-pole. Therefore, $\delta(d):=$ $\nu \geq 1$, where $\nu$ is the number of unstable poles of $\mathbf{P}$.

Suppose that $p_{i} \in \mathbb{C}_{+}, i=1, \ldots, \nu$ are the $\mathbb{C}_{+}$-poles of $\mathbf{P}$, ordered as follows: The first $k$ poles are at zero, where $0 \leq k \leq \nu$, The next $\ell$ of the $\mathbb{C}_{+}$-poles are real, $0 \leq \ell \leq$ $(\nu-k)$. The remaining $\mathbb{C}_{+}$-poles are $m$ complex-conjugate pairs $p_{i, i+1}=\Re e\left(p_{i}\right) \pm j \Im m\left(p_{i}\right)$, where $2 m=\nu-(k+\ell)$. Therefore, $d$ can be expressed as

$$
d(s)=s^{k} \prod_{i=k+1}^{k+\ell}\left(s-p_{i}\right) \prod_{i=k+\ell+1}^{k+\ell+m}\left(s^{2}+2 \alpha_{i} s+\omega_{i}^{2}\right) .
$$

where $\alpha_{i}:=\Re e\left(p_{i}\right)>0$ and $\omega_{i}:=\left|p_{i}\right|$ for complex conjugate poles. For $\beta_{i} \geq 0, i=1, \ldots, \nu$, define

$$
\chi_{\beta}(s):=\prod_{i=1}^{\nu}\left(s+\beta_{i}+\left|p_{i}\right|\right) .
$$

Lemma 1: Suppose that $p_{i} \in \mathbb{C}_{+}, i=1, \ldots, \nu$. Let $\beta_{0}>$ 0 and $\beta_{i} \geq 0$ be real constants satisfying $\left(\beta_{i}+\left|p_{i}\right|\right)>0$, $i=1, \ldots, \nu$. With $d(s)$ and $\chi_{\beta}(s)$ defined as in (18)-(19), the following norm equalities hold:

$$
\begin{gathered}
\left\|s\left(1-\frac{d(s)}{\chi_{\beta}(s)}\right)\right\|=\sum_{i=1}^{\nu}\left(\beta_{i}+p_{i}+\left|p_{i}\right|\right)=: \psi \\
\left\|s\left(1-\frac{s d(s)}{\left(s+\beta_{0}\right) \chi_{\beta}(s)}\right)\right\|=\beta_{0}+\psi
\end{gathered}
$$

The main result of the paper given below proposes a controller design method to improve the DM over an existing stabilizing controller, without changing its poles at $s=0$.

Proposition 2: DM improvement for unstable plants.

Suppose that $\mathbf{P} \notin \mathcal{S}$. Let $p_{i} \in \mathbb{C}_{+}, i=1, \ldots, \nu$, be the $\mathbb{C}_{+}$-poles of $\mathbf{P}$, where these poles are ordered as in (18).

a) Let $\mathbf{C}_{o}$ be a stabilizing controller for $\mathbf{P}$ such that $H_{o}=$ $\mathbf{P C}_{o}\left(1+\mathbf{P} \mathbf{C}_{o}\right)^{-1}$ is strictly proper. Then the controller $\mathbf{C}_{o}$ stabilizes $e^{-s h} \mathbf{P}$ for all $h \in\left[0, \tau_{m}\right)$, where

$$
\tau_{m}:=\left\|s H_{o}\right\|^{-1} \text {. }
$$

b) Let $\mathbf{C}_{o}$ be as in part a). Define $W$ as

$$
W(s):= \begin{cases}\frac{s}{s+\beta_{0}}, & \text { if } k=0 \\ 1, & \text { if } k \neq 0\end{cases}
$$

where $\beta_{0}>0$. For $i=1, \ldots, k$, choose $\beta_{i}>0$, and for $i=k+1, \ldots, \nu$, choose $\beta_{i} \geq 0$. Define $U:=W d / \chi_{\beta}$ and let

$$
\mathbf{C}_{\beta}=(1-U)\left(1+U \mathbf{C}_{o} \mathbf{P}\right)^{-1} \mathbf{C}_{o}
$$

With $\mathbf{C}_{\beta}$, the closed-loop input-output transfer function is

$$
H_{\beta}:=\mathbf{P C}_{\beta}\left(1+\mathbf{P C}_{\beta}\right)^{-1}=(1-U) H_{o} .
$$

Then the controller $\mathbf{C}_{\beta}$ in (24) stabilizes $e^{-s h} \mathbf{P}$ for $h \in$ $\left[0, \tau_{\beta m}\right)$, where $\tau_{\beta m}$ given by (26) is a lower bound on the delay margin:

$$
\tau_{\beta m}=\left\|s H_{\beta}\right\|^{-1}
$$

Furthermore, the delay margin lower bound satisfies

$$
\tau_{\beta m} \geq\left(\sum_{i=\varsigma}^{\nu} \beta_{i}+\sum_{i=1}^{\nu}\left(p_{i}+\left|p_{i}\right|\right)\right)^{-1}\left\|H_{o}\right\|^{-1}
$$

where $\varsigma=0$ if $k=0$ and $\varsigma=1$ if $k \neq 0$. A sufficient condition for the delay margin lower bound $\tau_{\beta m}$ to exceed the previous delay margin lower bound $\tau_{m}$ is

$$
\left(\sum_{i=k+1}^{\nu}\left(p_{i}+\left|p_{i}\right|\right)\right) \frac{\left\|H_{o}\right\|}{\left\|s H_{o}\right\|}<1 \text {. }
$$


If (28) holds, then choose $\beta_{i}$ as follows: If $k=0$, choose $\beta_{0}>0$; otherwise, choose $\beta_{0}=0$. For $i=1, \ldots, k$, choose $\beta_{i}>0$, and for $i=k+1, \ldots, \nu$ choose $\beta_{i} \geq 0$ such that

$$
\sum_{i=\varsigma}^{\nu} \beta_{i}<\frac{\left\|s H_{o}\right\|}{\left\|H_{o}\right\|}-\left(\sum_{i=k+1}^{\nu}\left(p_{i}+\left|p_{i}\right|\right)\right) \text {. }
$$

Then we have $\tau_{\beta m}>\tau_{m}$.

A special case of Proposition 2 is when the only $\mathbb{C}_{+}$-poles of $\mathbf{P}$ are at $s=0$ as stated in Corollary 1. These types of plants are of special interest in various applications, [6].

Corollary 1: Plants with a chain of integrators:

Suppose that $\mathbf{P} \notin \mathcal{S}$. In (18), let $d(s)=s^{\nu}$. Let $\mathbf{C}_{o}$ be a stabilizing controller for $\mathbf{P}$ such that $H_{o}=\mathbf{P C}_{o}(1+$ $\left.\mathbf{P} \mathbf{C}_{o}\right)^{-1}$ is strictly proper. Then by Proposition 2-(a), the controller $\mathbf{C}_{o}$ stabilizes $e^{-s h} \mathbf{P}$ for all $h \in\left[0, \tau_{m}\right)$, where $\tau_{m}=\left\|s H_{o}\right\|^{-1}$. For $i=1, \ldots, \nu$, choose $\beta_{i}>0$. Let

$\mathbf{C}_{\beta}=\left(1-\frac{s^{\nu}}{\prod_{i=1}^{\nu}\left(s+\beta_{i}\right)}\right)\left(1+\frac{s^{\nu}}{\prod_{i=1}^{\nu}\left(s+\beta_{i}\right)} \mathbf{C}_{o} \mathbf{P}\right)^{-1} \mathbf{C}_{o}$.

Then, the new complementary sensitivity is

$$
H_{\beta}=\mathbf{P C}_{\beta}\left(1+\mathbf{P} \mathbf{C}_{\beta}\right)^{-1}=\left(1-\frac{s^{\nu}}{\prod_{i=1}^{\nu}\left(s+\beta_{i}\right)}\right) H_{o} .
$$

i) The controller $\mathbf{C}_{\beta}$ in (30) stabilizes $e^{-s h} \mathbf{P}$ for $h \in$ $\left[0, \tau_{\beta m}\right)$, where $\tau_{\beta m}=\left\|s H_{\beta}\right\|^{-1}$. Furthermore,

$$
\tau_{\beta m} \geq\left(\sum_{i=1}^{\nu} \beta_{i}\right)^{-1}\left\|H_{o}\right\|^{-1} .
$$

A sufficient condition for $\tau_{\beta m}$ to exceed $\tau_{m}$ is the choice of $\beta_{i} \in \mathbb{R}_{+}, i=1, \ldots, \nu$ such that

$$
\sum_{i=1}^{\nu} \beta_{i}<\frac{\left\|s H_{o}\right\|}{\left\|H_{o}\right\|}
$$

ii) For any given delay $h=\tau \in \mathbb{R}_{+}$, the controller $\mathbf{C}_{\beta}$ in (30) can be designed to stabilize $e^{-s \tau} \mathbf{P}$ by choosing $\beta_{i} \in$ $\mathbb{R}_{+}, i=1, \ldots, \nu$, to satisfy

$$
\sum_{i=1}^{\nu} \beta_{i}<\tau^{-1}\left\|H_{o}\right\|^{-1}
$$

Furthermore, once $\beta_{i} \in \mathbb{R}_{+}$, are chosen, the corresponding lower bound $\tau_{\beta m}=\left\|s H_{\beta}\right\|^{-1}$ can be found, where $\tau_{\beta m} \geq$ $\tau$. Therefore, the controller $\mathbf{C}_{\beta}$ in (30) also stabilizes $e^{-s h} \mathbf{P}$ for all $h \in\left[0, \tau_{\beta m}\right)$, where $\tau_{\beta m} \geq \tau$.

Example 2: Plant with double integrator: Consider

$$
\mathbf{P}(s)=\frac{n(s)}{d(s) d_{s}(s)}=\frac{\left(s^{2}+16\right)}{s^{2}(s+4)} .
$$

Since $\mathbf{P}$ is strictly proper, the transfer function $H_{o}$ is strictly proper for every stabilizing controller $\mathbf{C}_{o}$. The first order controller $\mathbf{C}_{o}$ given in (36) below stabilizes $\mathbf{P}$ :

$$
\mathbf{C}_{o}=\frac{2(s+0.25)}{(s+5)} .
$$

a) The controller $\mathbf{C}_{o}$ is guaranteed to stabilize $e^{-s h} \mathbf{P}$ for all $h \in\left[0, \tau_{m}\right)$, where

$$
\tau_{m}=\left\|s H_{o}\right\|^{-1}=0.5 s
$$

(the actual DM is $0.6056 \mathrm{~s}$ ).

b) Using $\mathbf{C}_{o}$ given in (36), for the delay-free closed-loop transfer function we have $\left\|H_{o}\right\|=1.2969$ and $\left\|s H_{o}\right\|=2$. So, by choosing $\chi_{\beta}=\left(s+\beta_{1}\right)\left(s+\beta_{2}\right)$ with

$$
\left(\beta_{1}+\beta_{2}\right)<\frac{2.0}{1.3}=1.54
$$

we can have $\tau_{\beta m} \geq \tau_{m}$. For example, with $\beta_{1}=0.5, \beta_{2}=$ 0.75 , the controller (24) becomes

$$
\mathbf{C}_{\beta}=\frac{2.5(s+4)(s+0.25)}{(s+9.525)\left(s^{2}+2.426 s+5.442\right)} .
$$

This leads to a new DM lower bound

$$
\tau_{\beta m}=\left\|s H_{\beta}\right\|^{-1}=0.75 s
$$

(with the actual DM of $0.98 \mathrm{~s}$ ). The controller $\mathbf{C}_{\beta}$ in (37) stabilizes $e^{-s h} \mathbf{P}$ for $h \in\left[0, \tau_{\beta m}\right)$. Figure 2 shows $y(t)$ for a unit-step input at $u(t)$ with $\mathbf{C}_{o}$ (closed-loop is $H_{o}$ ) and $C_{\beta}$ (closed-loop is $H_{\beta}$ ).

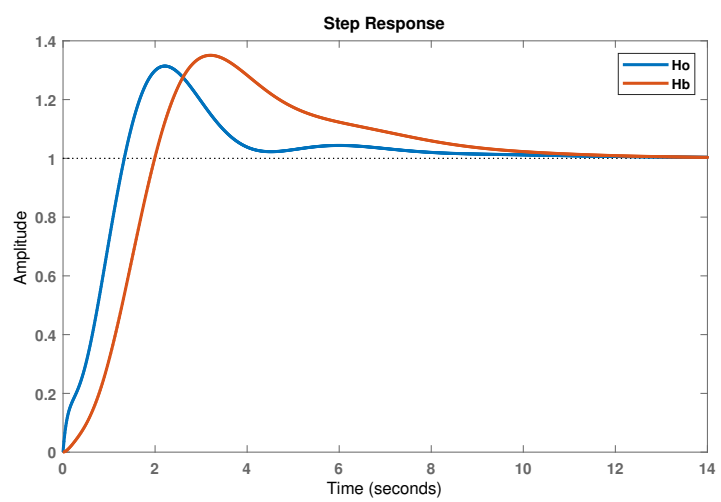

Fig. 2. Closed-loop step response of Example 2 for $h=0$, with $\mathbf{C}_{o}\left(H_{o}\right)$ and $\mathbf{C}_{\beta}\left(H_{\beta}\right)$.

The trade-off for delay margin improvement by using $\mathbf{C}_{\beta}$ is seen by comparing the step responses for $H_{o}$ and $H_{\beta}$ in Figure 2. Increasing the $D M$ has also increased the settling time from about $8 \mathrm{~s}$ to $10 \mathrm{~s}$.

\section{CONCLUSIONS}

In this paper we proposed a method to modify an initially designed stabilizing controller to improve a lower bound of the delay margin. The initial controller is assumed to be designed in such a way that steady-state tracking performance objectives are met. The modified controller is obtained by introducing some parameters, $\beta_{0}, \ldots, \beta_{\nu}$, where $\nu$ is the number of unstable poles of the plant. The modified controller order is $(\nu+1)$ higher than the initial controller order. The freedom in the design parameters affect the speed of the step response, and hence there can be additional optimization constraints in this design.

Detailed proofs and additional illustrative examples are given in the full version of the paper [3]. 


\section{REFERENCES}

[1] R. C. Dorf, and R. H. Bishop, Modern Control Systems, 13th Edition, Pearson, 2017.

[2] J. C. Doyle, B. A. Francis, and A. R. Tannenbaum, Feedback Control Theory, Macmillan, New York, 1992.

[3] A.N. Gündeş, and H. Özbay, "A controller tuning method for delay margin improvement," submitted for publication, 2019.

[4] P. Ju and H. Zhang, "Further results on the achievable delay margin using LTI control," IEEE Transactions on Automatic Control, vol. 61, pp. 3134-3139, 2016.

[5] R. H. Middleton, and D. E. Miller, "On the achievable delay margin using LTI control for unstable plants," IEEE Transactions on Automatic Control, vol. 52, pp. 1194-1207, 2007.

[6] S-I. Niculescu and W. Michiels, "Stabilizing a chain of integrators using multiple delays," IEEE Transactions on Automatic Control, vol. 49, pp. 802-807, 2004.

[7] H. Özbay, Introduction to Feedback Control Theory, CRC Press LLC, Boca Raton FL, 1999.

[8] H. Özbay, S. Gümüşsoy, K. Kashima, Y. Yamamoto, Frequency Domain Techniques for $H_{\infty}$ Control of Distributed Parameter Systems, SIAM, 2018.

[9] T. Qi, J. Zhu and J. Chen, "Fundamental limits on uncertain delays: When is a delay system stabilizable by LTI controllers?" IEEE Transactions on Automatic Control, vol. 62 No 3, pp. 1314-1328, 2017.

[10] J. Zhu, T. Qi, D. Ma, and J. Chen, Limits of Stability and Stabilization of Time-Delay Systems, A Small Gain Approach, Advances in Delays and Dynamics, Springer, Cham 2018. 